\title{
Measurements of beauty-decay electrons in ALICE at the LHC
}

\author{
Minjung Kim for the ALICE Collaboration ${ }^{1, a}$ \\ ${ }^{1}$ Inha University, Incheon, Republic of Korea
}

\begin{abstract}
Heavy quarks are essential probes of the evolution of the Quark-Gluon Plasma (QGP) created in ultra-relativistic heavy-ion collisions. In particular, the dependencies of the medium-induced parton energy loss on the parton mass and colour charge can be investigated via measurements of open heavy-flavour hadrons. To quantify medium effects in AA collisions, one needs to study p-A collisions in order to disentangle possible cold nuclear matter effects. Thanks to the excellent vertex and impact parameter resolution of ITS and electron-identification capability provided by TPC and TOF in the ALICE experimental setup, measurements of beauty production in $\mathrm{pp}, \mathrm{p}-\mathrm{Pb}$ and $\mathrm{Pb}-\mathrm{Pb}$ collisions could be done via electrons from semi-leptonic decays of beauty hadrons. The recent measurements of beauty-decay electrons in $\mathrm{p}-\mathrm{Pb}$ collisions at $\sqrt{s_{\mathrm{NN}}}=5.02 \mathrm{TeV}$ and in $\mathrm{Pb}-\mathrm{Pb}$ collisions at $\sqrt{s_{\mathrm{NN}}}=2.76 \mathrm{TeV}$ in ALICE are presented.
\end{abstract}

\section{Introduction}

In ultra-relativistic heavy-ion collisions, heavy quarks (charm and beauty) are predominantly created in the initial hard scattering processes due to their large masses [1]. Since their production is expected to be earlier than the formation of the Quark-Gluon Plasma (QGP), heavy quarks can traverse the hot and dense medium created in heavy-ion collisions, interacting with its constituents and experience the full system evolution. The medium-induced parton energy loss of quarks is smaller than that of gluons due to their smaller colour coupling to the medium with respect to gluons. Furthermore, the medium-induced parton energy loss of heavy partons is expected to be suppressed compared to light partons in both radiative energy loss process [2-4] and collisional energy loss process [5]. The energy loss hierarchy among different parton species can be studied via measurements of open heavy-flavour hadrons nuclear modification factor $\left(R_{\mathrm{AA}}\right)$ and comparison with those of light-flavour hadrons. $R_{\mathrm{AA}}$ is defined as :

$$
R_{\mathrm{AA}}=\frac{1}{<T_{\mathrm{AA}}}>\frac{\mathrm{d} N_{\mathrm{AA}} / \mathrm{d} p_{\mathrm{T}}}{\mathrm{d} \sigma_{\mathrm{pp}} / \mathrm{d} p_{\mathrm{T}}},
$$

where $\mathrm{d} N_{\mathrm{AA}} / \mathrm{d} p_{\mathrm{T}}$ is the $p_{\mathrm{T}}$-differential yield in AA collisions, $\mathrm{d} \sigma_{\mathrm{pp}} / \mathrm{d} p_{\mathrm{T}}$ is the $p_{\mathrm{T}}$-differential cross section in pp collisions, and $<T_{\mathrm{AA}}>$ is the average nuclear overlap function for a given centrality class in AA collisions. Especially, separate measurements of beauty hadrons from charm hadrons can test the mass dependence of medium-induced parton energy loss even at high $p_{\mathrm{T}}$ [6].

To quantify medium effects in AA collisions through the $R_{\mathrm{AA}}$, one needs to disentangle the possible cold nuclear matter (CNM) effects, such as the modification of parton distribution functions in the

\footnotetext{
a e-mail: minjung.kim@cern.ch
} 

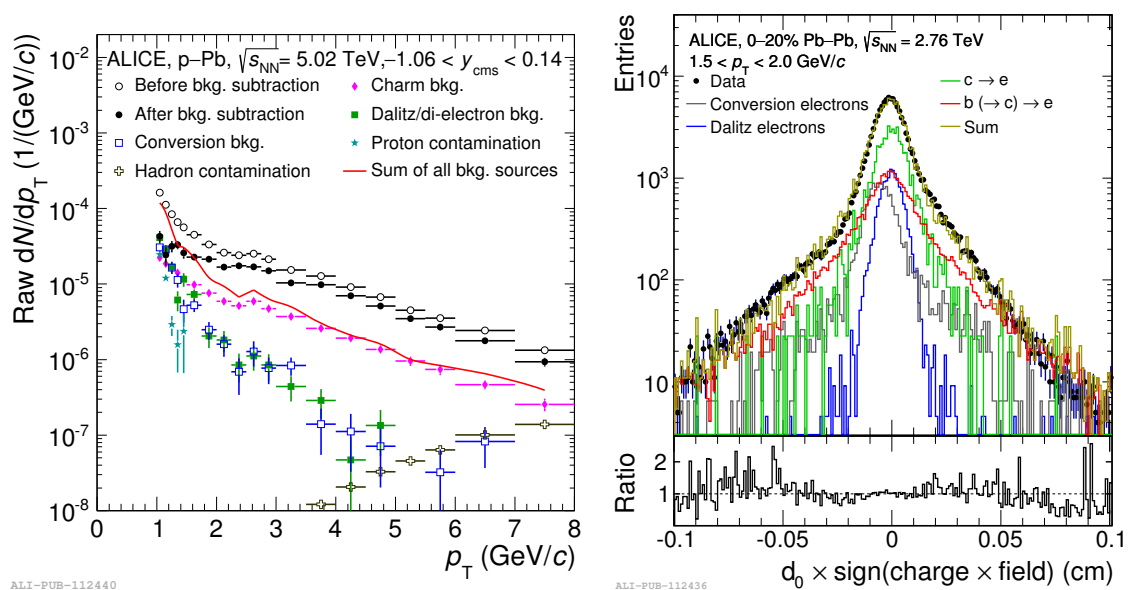

Figure 1. Raw $p_{\mathrm{T}}$ distributions of electrons after applying elD and minimum $d_{0}$ cut compared with background components in $\mathrm{p}-\mathrm{Pb}$ collisions (left). $d_{0}$ distributions of electrons in the $20 \%$ most central $\mathrm{Pb}-\mathrm{Pb}$ collisions (right). The individual distributions for each sources were obtained from Monte Carlo simulations with datadrive corrections [12]

nucleus [7] and the transverse momentum broadening from multiple scattering [8], by measuring the nuclear modification factor in $\mathrm{p}-\mathrm{A}$ collisions $\left(R_{\mathrm{pA}}\right)$.

ALICE (A Large Ion Collider Experiment) is dedicated to studying QGP properties [9]. The ALICE detector provides electron identification capability down to very low $p_{\mathrm{T}}$ and excellent momentum and track impact parameter resolution at mid-rapidity region [10, 11]. Beauty-hadron production at mid-rapidity have been measured via electrons from semi-leptonic decays of beauty hadrons.

\section{Analysis}

In the mid-rapidity region, charged particles were reconstructed with Inner Tracking System (ITS), which consists of the 6-layered silicon detector and Time Projection Chamber (TPC) embedded in a magnetic field of $0.5 \mathrm{~T}$, providing $p_{\mathrm{T}}$ resolution better than $2.5 \%$ for $p_{\mathrm{T}} \leq 30 \mathrm{GeV} / c$ and transverse impact parameter $\left(d_{0}\right)$ resolution better than $75 \mu \mathrm{m}$ for $p_{\mathrm{T}}>1 \mathrm{GeV} / c$ [13]. $d_{0}$ is defined as the track distance of closest approach to the primary vertex in the plane transverse to the beam axis. Electron identification (eID) was performed by requiring the $\mathrm{dE} / \mathrm{dx}$ in the TPC to be within $-0.5 \sigma$ and $+3 \sigma$ with respect to the expected $\mathrm{dE} / \mathrm{dx}$ of electrons in both $\mathrm{p}-\mathrm{Pb}$ and $\mathrm{Pb}-\mathrm{Pb}$ analyses. The remaining hadron contamination was determined after requiring the measured time-of-flight in Time Of Flight (TOF) detector to be within $\pm 3 \sigma$ of the expected value for electrons for whole measured $p_{\mathrm{T}}$ range in the $\mathrm{Pb}-\mathrm{Pb}$ analysis and for $p_{\mathrm{T}} \geq 2.5 \mathrm{GeV} / c$ in the $\mathrm{p}-\mathrm{Pb}$ analysis for further hadron rejection [12].

The electron candidates fulfilling above selection criteria are composed of signal, i.e. beauty-decay electrons, and background electrons coming from various sources [14]. At low $p_{\mathrm{T}}$, the dominant sources of background electrons are Dalitz decays of neutral light mesons, di-electron decays of lightvector mesons and photon conversions in the beam pipe and inner most layer of ITS. At high $p_{\mathrm{T}}$, semi-leptonic decays of charm hadrons are the main background source. Long lifetime of beauty hadrons leads to larger $d_{0}$ of electrons from beauty-hadron decays than those of background electrons. Based on impact parameter information, two different analysis strategies were used. 
In $\mathrm{p}-\mathrm{Pb}$ analysis, requirement of minimum $d_{0}$ for electron candidates was used for extracting the signal from electron candidates, which was successfully applied in previous measurements in pp collisions at $\sqrt{s}=7 \mathrm{TeV}$ and $\sqrt{s}=2.76 \mathrm{TeV}[14,15]$. Electron candidates satisfying minimum $d_{0}$ are shown as open circle spectrum as a function of $p_{\mathrm{T}}$ in left panel of Figure 1. The remaining background electrons were estimated by Monte Carlo simulations re-weighted to match the $p_{\mathrm{T}}$ distributions of the background sources in data using ALICE measurements [16-18]. The fraction of hadron contamination was calculated by parameterizing TPC $\mathrm{dE} / \mathrm{dx}$ at high $p_{\mathrm{T}}\left(p_{\mathrm{T}} \geq 4 \mathrm{GeV} / c\right)$ and ITS $\mathrm{dE} / \mathrm{dx}$ at low $p_{\mathrm{T}}\left(1 \mathrm{GeV} / c<p_{\mathrm{T}}<1.2 \mathrm{GeV} / c\right)$. By subtracting background components from electron candidates, the raw yield of beauty-decay electrons shown in left panel of Figure 1 as black-solid circle spectrum was obtained.

In $\mathrm{Pb}-\mathrm{Pb}$ collisions, the signal extraction was done by fitting $d_{0}$ distribution of electron candidates with signal (beauty-decay electrons) and background templates obtained from Monte Carlo simulations as shown in right panel of Figure 1. The discrepancy of $d_{0}$ distributions between data and Monte Carlo simulations caused by difference of resolution and background-source spectra was corrected based on data-driven method and ALICE measured D meson spectrum [19], respectively. The remaining imperfection of Monte Carlo simulations on describing data was propagated to final systematic uncertainty [12]. Since both the hadron contamination and Dalitz electrons originate from or close to interaction point, their $d_{0}$ distributions are similar. Thus, the contribution of hadron contamination was absorbed into Dalitz electrons.

After correction for geometrical acceptance as well as efficiency of the track reconstruction, matching and track selection criteria, the $p_{\mathrm{T}}$-differential spectrum of electrons from beauty-hadron decays could be obtained in $\mathrm{p}-\mathrm{Pb}$ collisions and in $\mathrm{Pb}-\mathrm{Pb}$ collisions. In order to calculate $R_{\mathrm{pPb}}$ as well as $R_{\mathrm{PbPb}}$, corresponding pp reference spectra at $\sqrt{s}=5.02 \mathrm{TeV}$ and $\sqrt{s}=2.76 \mathrm{TeV}$, respectively, was obtained by a pQCD-driven $\sqrt{s}$-scaling of the cross section of electrons from beauty-hadron decays measured in pp collisions at $\sqrt{s}=7 \mathrm{TeV}[14]$.

\section{Results}

The nuclear modification factor of electrons from beauty-hadron decays in $\mathrm{p}-\mathrm{Pb}$ collisions $\left(R_{\mathrm{pPb}}\right)$ and in the $20 \%$ most central $\mathrm{Pb}-\mathrm{Pb}$ collisions $\left(R_{\mathrm{PbPb}}\right)$, measured as a function of $p_{\mathrm{T}}$, is shown in Figure 2. The $R_{\mathrm{pPb}}$ is compatible with unity within uncertainties for measured $p_{\mathrm{T}}$ range. The result is well-described by theoretical model calculations including CNM effects and blast-wave model calculations considering possible hydrodynamic effects [20]. The measured $R_{\mathrm{PbPb}}$ is described by theoretical models of beauty-quark energy loss in the QGP within uncertainties. Thus, the suppression of electrons from beauty-hadron decays in the $20 \%$ most central $\mathrm{Pb}-\mathrm{Pb}$ collisions for $p_{\mathrm{T}}>3 \mathrm{GeV} / c$ can be understood as an effect of the hot and dense medium created in $\mathrm{Pb}-\mathrm{Pb}$ collisions on beauty quarks.

\section{Acknowledgements}

This work was supported by National Research Foundation of Korea (NRF), Basic Science Research Program through the National Research Foundation of Korea funded by the Ministry of Education, Science and Technology (Grant number: 2014R1A1A1008246) and by Inha University Research Grant (INHA-47297).

\section{References}

[1] A. Andronic et al., Eur. Phys. J. C76, 107 (2016), arXiv:1506.03981

[2] Y.L. Dokshitzer, D.E. Kharzeev, Phys. Lett. B519, 199 (2001), hep-ph/0106202 

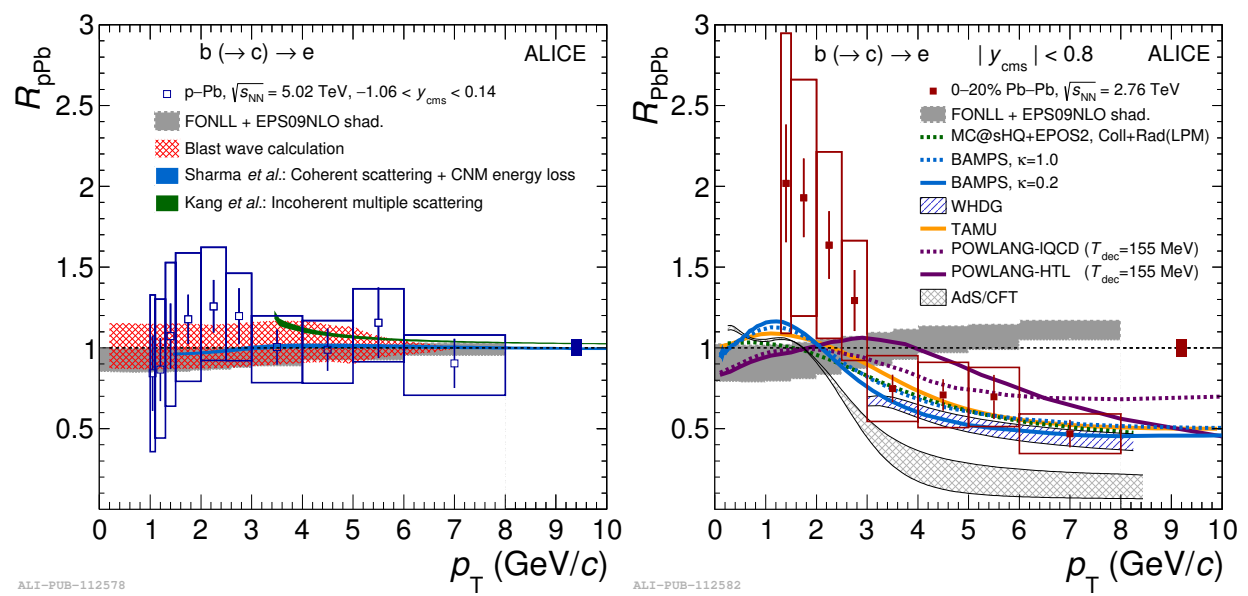

Figure 2. Nuclear modification factor of electrons from beauty-hadron decays at mid-rapidity in $\mathrm{p}-\mathrm{Pb}$ collisions at $\sqrt{s_{\mathrm{NN}}}=5.02 \mathrm{TeV}$ (left) and in $\mathrm{Pb}-\mathrm{Pb}$ collisions at $\sqrt{s_{\mathrm{NN}}}=2.76 \mathrm{TeV}$ in the $20 \%$ most central collisions (right) with several theoretical model predictions [12]

[3] M. Djordjevic, M. Gyulassy, Nucl. Phys. A733, 265 (2004), nucl-th/0310076

[4] N. Armesto, C.A. Salgado, U.A. Wiedemann, Phys. Rev. D69, 114003 (2004), hep-ph/0312106

[5] H. van Hees, V. Greco, R. Rapp, Phys. Rev. C73, 034913 (2006), 0508055

[6] N. Armesto, A. Dainese, C.A. Salgado, U.A. Wiedemann, Phys. Rev. D 71, 054027 (2005)

[7] K.J. Eskola, H. Paukkunen, C.A. Salgado, JHEP 04, 065 (2009), arXiv:0902.4154

[8] I. Vitev, Phys. Rev. C 75, 064906 (2007)

[9] K. Aamodt et al., (ALICE Collaboration), JINST 3, S08002 (2008)

[10] F. Carminati et al., (ALICE Collaboration), J. Phys. G: Nucl. Part. Phys. 30, 1517 (2004)

[11] B. Alessandro et al., (ALICE Collaboration), J. Phys. G: Nucl. Part. Phys. 32, 1295 (2004)

[12] J. Adam et al., (ALICE Collaboration) (2016), arXiv:1609.03898

[13] B. Abelev et al., (ALICE Collaboration), Int. J. Mod. Phys. A29, 1430044 (2014), arXiv: 1402.4476

[14] B. Abelev et al., (ALICE Collaboration), Phys. Lett. B721, 13 (2013), arXiv:1208.1902

[15] B. Abelev et al., (ALICE Collaboration), Phys. Lett. B738, 97 (2014), arXiv:1405.4144

[16] B. Abelev et al., (ALICE Collaboration), Phys. Lett. B728, 25 (2014), arXiv:1307.6796

[17] J. Adam et al., (ALICE Collaboration) (2016), arXiv:1601.03658

[18] B. Abelev et al., (ALICE Collaboration), Phys. Rev. Lett. 113, 232301 (2014), arXiv:1405.3452

[19] B. Abelev et al., (ALICE Collaboration), JHEP 09, 112 (2012), arXiv:1203.2160

[20] A.M. Sickles, Phys. Lett. B731, 51 (2014), arXiv:1309.6924 